\title{
Dead layer thickness estimation at the ferroelectric film-metal interface in PZT
}

Cite as: Appl. Phys. Lett. 114, 132902 (2019); https://doi.org/10.1063/1.5084019

Submitted: 03 December 2018 . Accepted: 16 March 2019. Published Online: 02 April 2019

Yu. V. Podgorny, K. A. Vorotilov, A. S. Sigov, and J. F. Scott (D)
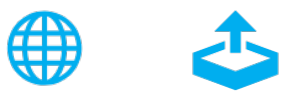

\section{ARTICLES YOU MAY BE INTERESTED IN}

A critical review of recent progress on negative capacitance field-effect transistors

Applied Physics Letters 114, 090401 (2019); https://doi.org/10.1063/1.5092684

Ferroelectric polarization-controlled resistive switching in $\mathrm{BaTiO}_{3} / \mathrm{SmNiO}_{3}$ epitaxial heterostructures

Applied Physics Letters 114, 102901 (2019); https://doi.org/10.1063/1.5066032

Coexistence of dielectric relaxation and magnetic relaxation in compressively strained $\mathrm{BiFeO}_{3} / \mathrm{BaO}_{0} \mathrm{SrO} . \mathrm{TiO}_{3}$ superlattices

Applied Physics Letters 114, 112902 (2019); https://doi.org/10.1063/1.5085350

\section{Applied Physics Reviews} Now accepting original research 


\title{
Dead layer thickness estimation at the ferroelectric film-metal interface in PZT
}

Cite as: Appl. Phys. Lett. 114, 132902 (2019); doi: 10.1063/1.5084019

Submitted: 3 December 2018 . Accepted: 16 March 2019 .

Published Online: 2 April 2019

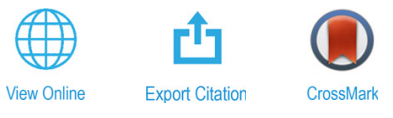

Yu. V. Podgorny, ${ }^{\top}$ K. A. Vorotilov, A. S. Sigov, ${ }^{\top}$ and J. F. Scott ${ }^{2, a)}$ (D)

\author{
AFFILIATIONS \\ ${ }^{7}$ MIREA - Russian Technological University, Moscow 119454, Russia \\ ${ }^{2}$ Schools of Chemistry and Physics, St. Andrews University, St. Andrews KY16 9SS, United Kingdom
}

${ }^{a)}$ Author to whom correspondence should be addressed: jfs4@st-andrews.ac.uk

\begin{abstract}
Different models for estimation of the dead layer thickness at the ferroelectric film-metal interface are discussed, including the small-signal capacitance model and two methods based on dielectric hysteresis analysis-one based on slopes of the hysteresis loops at the coercive field and the other method based on comparison of dielectric hysteresis portraits. It is shown that the latter technique yields more reliable data as it excludes the effect of leakage and relaxation loss. Conductivity may have a pronounced effect on the validity of dead-layer thickness data. This work relates peripherally to negative capacitance in ferroelectric films and to lozenge-shaped hysteresis curves.
\end{abstract}

Published under license by AIP Publishing. https://doi.org/10.1063/1.5084019

It has been observed that the film thickness of the ferroelectric material in metal-ferroelectric-metal structures has a profound impact on their dielectric and ferroelectric properties due to numerous possible reasons, including lattice distortion, defects (point defects, dislocations, etc.), and so-called "dead layers". ${ }^{1-10}$

As a rule, the thickness effects are studied by comparison of capacitance of samples with different ferroelectric film thicknesses. The thickness dependence of the effective dielectric constant can be obtained by assuming that a non-ferroelectric layer (dead layer) exists at the metal-ferroelectric interface. These measurements are typically performed at quite a high frequency $(\sim 100 \mathrm{kHz})$ and small-signal with amplitude of ten millivolts.

It is interesting to extract information about the dead layer thickness directly from the hysteresis loops. Tagantsev and co-authors theoretically investigated the switching of the multilayer structure "ferroelectric + thin dielectric layer".,10 These authors proposed to extract the dead layer value from the slope of the hysteresis loop at the coercive field or remnant polarization. Böttger and Waser used this method to determine a depolarization factor which contains quantitative information about interfacial layers as well as ferroelectrically passive zones in the bulk. ${ }^{11}$ Unfortunately, such parameters depend heavily on the leakage current and relaxation loss and, thus, on the measurement frequency. ${ }^{12-14}$ Hence, the obtained data may be invalid. As shown by Waser (Refs. 11-14), PUND (repetitively switching voltage polarity) does not eliminate the relaxation problem.

In the present work, we propose a dead layer estimation technique based on the dielectric portraits ${ }^{25}$ analysis. A comparative analysis with Tagantsev's and small-signal capacitance techniques is performed.

Dense and porous lead zirconate-titanate (PZT) films ( $\mathrm{Zr} / \mathrm{Ti}$ $=48 / 52$ ) were obtained by sol-gel techniques. Dense films were prepared by an alkoxide route; for details, see Ref. 15. Porous films were obtained by a polyvinylpyrrolidone (PVP) assisted process; for details, see Ref. 16. Silicon wafers with the Pt $(150 \mathrm{~nm}) / \mathrm{TiO}_{2}(10 \mathrm{~nm}) / \mathrm{SiO}_{2}$ $(300 \mathrm{~nm}) / \mathrm{Si}$ structure (produced by Inostec, Korea) were used as substrates. The thickness of the films was varied from $\sim 170 \mathrm{~nm}$ to $\sim 1200 \mathrm{~nm}$ in accordance with the number of deposited layers (from 5 to 30 layers). The porosity of porous films equals $19.3 \pm 2.8 \mathrm{vol}$. $\%$, as determined by ellipsometry in the visible (well below the optical bandgap and hence approximately wavelength-independent) and the Lorentz-Lorenz model. Finally, we have obtained XRD 2-theta graphs for the samples studied here. These are as expected, showing only a single phase and with peaks at 2-theta compatible with the Bragg scattering calculated from the literature for the nominal Ti/Zr ratios.

Electrical measurements were performed with the use of a mercury probe with the diameter of $\sim 760 \mu \mathrm{m}$ to obtain an upper contact to the film (MCD 802-150, MDC Corp.). Ferroelectric hysteresis was measured at the frequency of $100 \mathrm{~Hz}$ (TF Analyzer 2000E, AixACCT). The capacitance-voltage $C(V)$ dependences were obtained at the frequency of $100 \mathrm{kHz}$ with an oscillation voltage of $100 \mathrm{mV}$ using a 4140B LCR meter (Agilent).

Polarization loops of dense and porous PZT films show different behaviors, as shown in Fig. 1. The dense films demonstrate asymmetric loops, and their coercive field $E_{c}$ depends on the thickness of the 


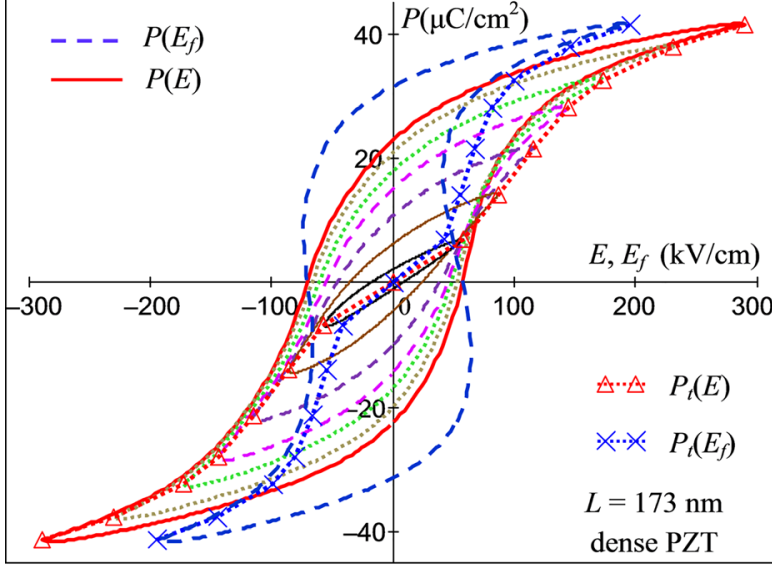

(a)

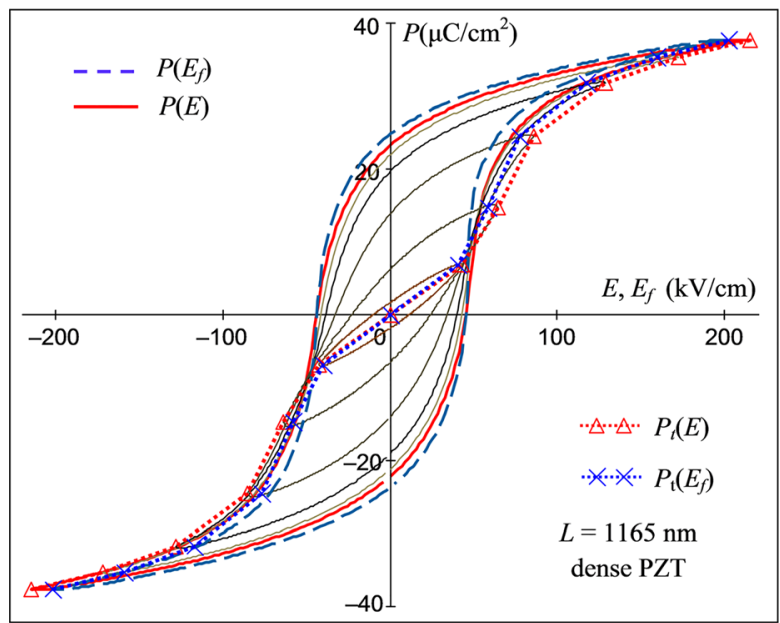

(b)

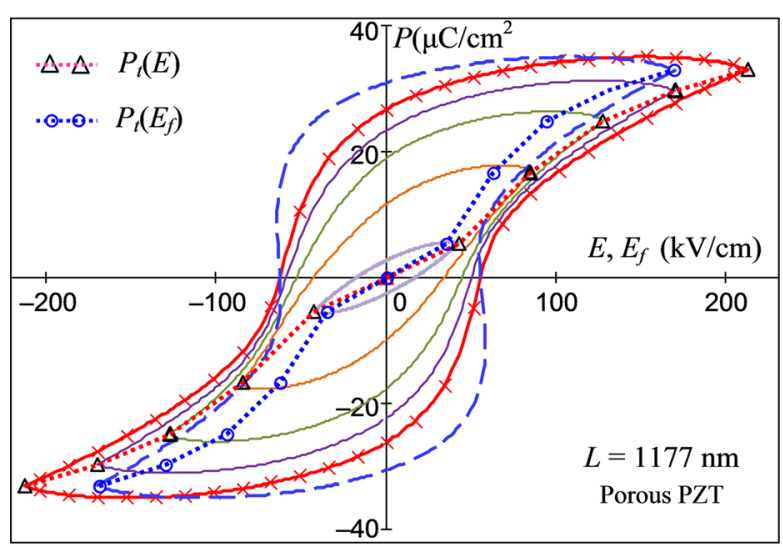

(c)

FIG. 1. $P(E)$ hysteresis loops (solid line) and dielectric portraits in the coordinates of the applied field $P_{t}(E)$ and the field inside the ferroelectric film volume $P_{t}\left(E_{f}\right)$ (dotted line) for different PZT films: the dense PZT film with the thickness of $173 \mathrm{~nm}$ (a), the dense PZT film with the thickness of $1067 \mathrm{~nm}(\mathrm{~b})$, and the porous PZT film (19 vol. \%) with the thickness of $1177 \mathrm{~nm}$ (c) film [Figs. 1(a) and 1(b)]; the observed hysteresis loops are asymmetric with respect to changing $+\mathrm{V}$ to $-\mathrm{V}$ applied voltage. This asymmetry does not disappear if the top and bottom electrodes are made of the same metal. A large number were measured, and we show here only two or three representative sets of data. In contrast to this, the coercive field of porous films $E_{c}$ has no pronounced thickness dependence [Fig. 1 (c)] (5 were measured without significant differences).

Some conclusions should take into account the hysteresis loops analysis. First, the measured values of the remnant polarization depend on the ohmic leakage current and the relaxation losses, which depend on the measurement frequency. In turn, their contribution is proportional to the applied voltage amplitude. At the same time, polarization values obtained at the loop tips are free from this dependence, ${ }^{12-14}$ and our applied voltages were sufficient to show almost complete saturation. Second, polarization values at the tips of the hysteresis loops tend to saturation with the voltage amplitude increase, regardless of the thickness of both the ferroelectric film and the dead layer. At first glance, the dielectric portrait cannot be useful to extract the dead layer thickness; however, such is not the case, as is shown below.

Figure 2 shows the dielectric portraits (or "profiles") of two ferroelectric structures with porous (19 vol. \%) PZT films with the thickness of 300 and $1177 \mathrm{~nm}$ for positive polarity of the applied voltage. The terms "portrait" and profile are not new technical terms but are used in their usual English-language sense to describe the shape of the $\mathrm{P}(\mathrm{E})$ ferroelectric hysteresis curves, particularly their tips at the highest $\mathrm{P}$ and $\mathrm{E}$ values. Like the portrait of a person, the profile describes a side-view: Are the nose and chin straight or curved? Sharp or rounded? Horizontal or droopy? Does the shape have an upward concave curvature or is it convex?

We take into account that at the same value of the polarization in the film volume, the corresponding values of the field in the ferroelectric volume $E_{f}$ should be the same regardless of the film thickness. The following relationship is valid for the two-layer capacitor: ${ }^{17}$

$$
E_{f}\left(P_{f}\right)=\left[\left(1+\frac{l_{d}}{L}\right) \frac{V}{L}-\frac{l_{d}}{L} \frac{P_{f}}{\varepsilon_{0} \varepsilon_{d}}\right] /\left(1+\frac{l_{d}}{L} \frac{\varepsilon_{f}}{\varepsilon_{d}}\right),
$$

where $L$ and $l_{d}$ are the thickness of the ferroelectric and the dead layer, respectively; $V$ is the applied voltage; $\varepsilon_{f}$ and $\varepsilon_{d}$ are the linear (optical) dielectric permittivity of the volume of the ferroelectric and the dead layer, respectively; and $P_{f}$ is the spontaneous polarization.

In the case of $l_{d} / L \ll 1$ and if the values of the optical permittivity $\varepsilon_{f}$ in the bulk of the film and the permittivity of the dead layer $\varepsilon_{d}$ are equal

$$
E_{f}\left(P_{t}\right) \approx \frac{V}{L}-\frac{l_{d}}{L} \frac{P_{t}}{\varepsilon_{0} \varepsilon_{d}},
$$

where $P_{t}$ is the polarization at the tip of the loop.

Dependence (2) makes it possible to compose an equation for any value of the field $E_{f}$ in the volume of each ferroelectric structure with identical ferroelectric films of different thicknesses $L_{1}$ and $L_{2}$

$$
\frac{V_{1}}{L_{1}}-\frac{l_{d}}{L_{1}} \frac{P_{t}}{\varepsilon_{0} \varepsilon_{d}}=\frac{V_{2}}{L_{2}}-\frac{l_{d}}{L_{2}} \frac{P_{t}}{\varepsilon_{0} \varepsilon_{d}},
$$

from which the dead layer thickness can be expressed as

$$
l_{d}=\frac{\varepsilon_{0} \varepsilon_{d}}{P_{t}} \frac{V_{2} L_{1}-V_{1} L_{2}}{L_{1}-L_{2}}=\frac{\varepsilon_{0} \varepsilon_{d}}{P_{t}}\left(E_{1}-E_{2}\right) \frac{L_{1} \cdot L_{2}}{L_{2}-L_{1}} .
$$



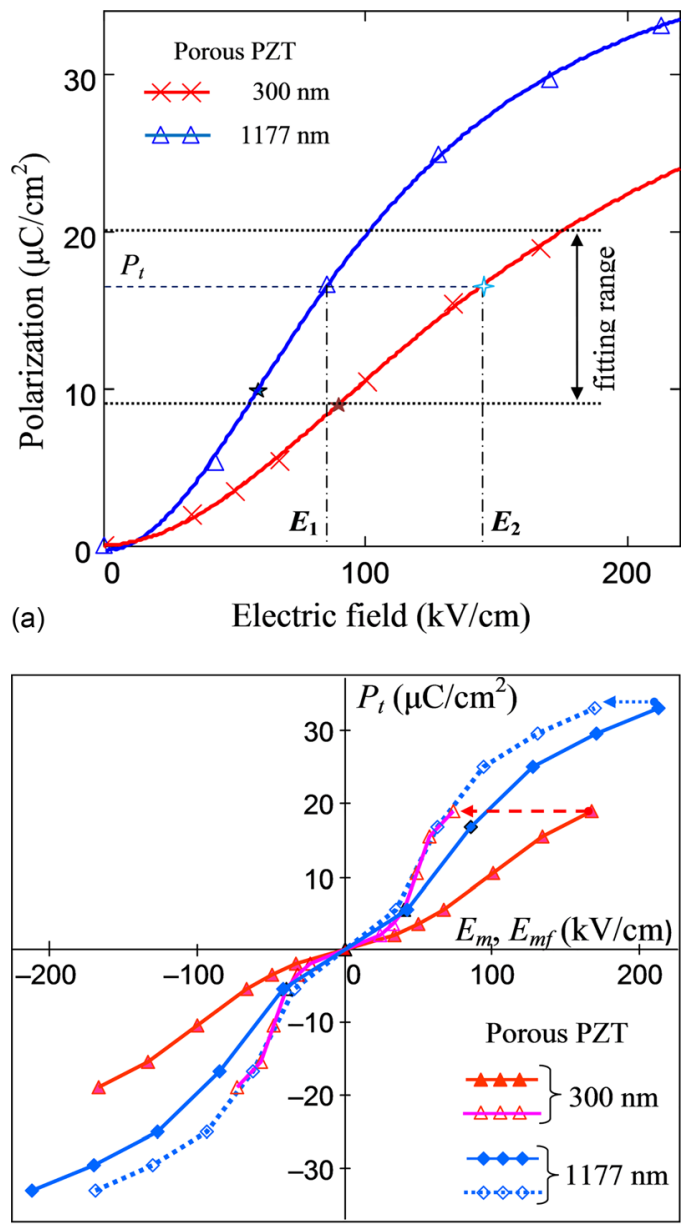

(b)

FIG. 2. Dead layer estimation techniques: (a) Dielectric portraits of porous PZT films with the thicknesses of 300 and $1177 \mathrm{~nm}$. The electric field values $E_{1}$ and $E_{2}$ correspond to the definite value of the polarization $P_{t}$ at the tips of the hysteresis loops. (b) Estimation of the dead layer thickness by an iteration technique.

According to Eq. (3), it is sufficient to determine the electric field values $E_{1}$ and $E_{2}$ for any definite polarization value $P_{t}$ using the corresponding dielectric portraits, see Fig. 2(a).

An alternative method for determining the equivalent dead layer thickness is to iterate the slopes of the dielectric $P_{t}\left(E_{f}\right)$ portraits of structures with different film thicknesses. The value of $l_{d}$ is gradually increased in Eq. (2) from zero until the dielectric portraits of two ferroelectric structures coincide in the region of their maximum steepness, as is shown in Fig. 2(b). Both methods yield the same results.

Table I shows the obtained values of the bulk permittivity $\varepsilon_{\mathrm{b}}$, the capacity $C_{d}$, and the dead layer thickness $l_{s s}$ obtained by the smallsignal capacitance technique. ${ }^{1-5,8}$ The optical permittivity of PZT films was obtained from ellipsometric measurements as $\varepsilon_{d}=n^{2}$.

In Table I, the dead layer thickness $l_{T g}$ was calculated from the slope of the hysteresis loops at $E_{\mathrm{c}}$ according to Tagantsev's technique, ${ }^{9-11}$ and the dead layer thickness $l_{\mathrm{Pt}}$ was obtained in accordance with the described polarization hysteresis tip method.
TABLE I. Dead layer data for dense and porous PZT films obtained by different techniques.

\begin{tabular}{lcccccc}
\hline \hline Porosity \% & $\varepsilon_{b}$ & $C_{d} \mu \mathrm{F} / \mathrm{cm}^{2}$ & $\varepsilon_{d}=n^{2}$ & $l_{s s} \AA$ & $l_{\mathrm{Tg}} \AA$ & $l_{\mathrm{Pt}} \AA$ \\
\hline 0 & 882 & 47 & 6.25 & 2.1 & 1.6 & 2.2 \\
19 & 659 & 6.9 & 4.57 & 12 & 3.8 & 5.8 \\
\hline \hline
\end{tabular}

It should be noted that the formulas used to calculate the thickness of the dead layer by the small-signal capacitance and Tagantsev's techniques are identical in shape. ${ }^{11}$ The main difference is that in the second case, the differential values of the permittivity at the coercive field $\left(d P / d E_{c}\right) / \varepsilon_{0}$ (in the region of the maximum steepness of the hysteresis loop) are used for the calculation instead of small-signal values. Additionally, as it was mentioned before, differential values have strong dependence on leakages and relaxation losses in the ferroelectric film in contrast to values obtained from small-signal measurements. Leakage current increases with the applied voltage and leads to an increase in hysteresis loop steepness. ${ }^{12-14}$ As a result, this technique may be in error.

Comparison of the dead layer thickness values obtained by the small-signal capacitance and the polarization tip techniques gives the same results for the dense film and sufficiently differs for the porous ones. The reason is high leakage currents in porous films changing the potential distribution across the layered structure. ${ }^{16}$

Figure 3 shows an equivalent circuit of the ferroelectric film with the dead layer, taking into account conductivity $\sigma$ of the ferroelectric film. A shunting effect of the conductivity $\sigma$ on the voltage distribution in the layered structure varies inversely with the square of the measurement frequency $\omega$

$$
\frac{V}{V_{b}}=1+\frac{l_{d}}{L} \frac{\varepsilon_{b}}{\varepsilon_{d}} \cdot\left[1-\frac{1}{2}\left(\frac{\sigma}{\omega \varepsilon_{0} \varepsilon_{b}}\right)^{2} \cdot\left(\frac{\varepsilon_{b}-\varepsilon_{d}}{\varepsilon_{d}}\right)^{2}\right] .
$$

Effective capacitance may be expressed as

$$
C_{e f f}(\sigma, \omega) \approx \frac{\varepsilon_{b} \varepsilon_{d} \varepsilon_{0}}{\varepsilon_{b} l+\varepsilon_{d} L} \cdot\left[1+\left(\frac{\sigma}{\omega \varepsilon_{0}}\right)^{2} \cdot \frac{1}{\varepsilon_{b} \varepsilon_{d}} \cdot \frac{L l \cdot\left(\varepsilon_{b}-\varepsilon_{d}\right)^{2}}{\left(\varepsilon_{b} l+\varepsilon_{d} L\right)^{2}}\right] .
$$

This equation shows that the shunting effect becomes more pronounced when the difference between the values of the permittivity of

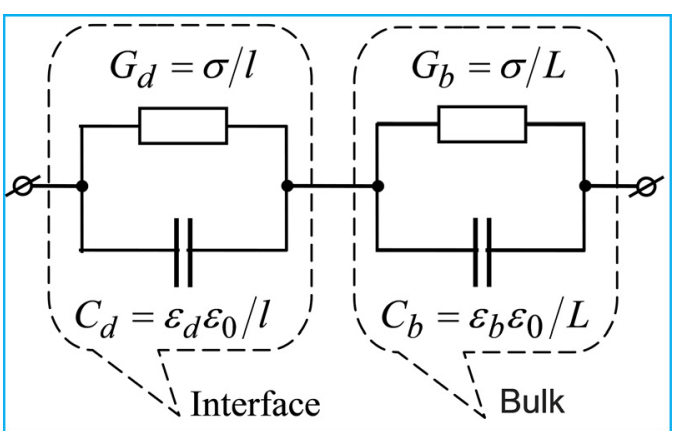

FIG. 3. Equivalent circuit of the ferroelectric structure with the dead layer and conductivity $\sigma$. The latter parameter assumes the same throughout the film thickness. 
the ferroelectric volume and the dead layer increases, and the measurement frequency decreases.

Figure 4 shows the dependence of error in capacitance $C_{b}$ of the ferroelectric volume of the porous PZT film as a function of the conductivity shunting the dead layer at the frequency of $100 \mathrm{~Hz}$. This error can be sufficiently high that conductivity should be taken into account at low frequency measurements.

Shunted equivalent conductivity $\sigma$ may be found as

$$
\sigma=\omega \varepsilon_{0} \varepsilon_{b} \cdot \sqrt{2\left(1-\frac{l_{P}}{l_{s s}}\right)} \cdot \frac{\varepsilon_{d}}{\varepsilon_{b}-\varepsilon_{d}} .
$$

According to the results in Table I, the value of the equivalent conductivity of the porous film at the frequency of $100 \mathrm{~Hz}$ calculated from Eq. (6) is $2.6 \mu \mathrm{S} / \mathrm{cm}$. Note that this value is higher by about two orders than the static conductivity values for porous PZT films ${ }^{16}$ due to the effect of low-frequency relaxation losses. ${ }^{18}$

It should be noted that the dead layer discussed here and the Schottky depletion layer are not the same thing, as is sometimes supposed. ${ }^{1-5}$ The depletion layer has low conductivity due to the absence of charge carriers. ${ }^{19}$ Moreover, its width is inversely proportional to the concentration of charge carriers, which seems contrary to the experimental results demonstrating a higher dead layer thickness in the porous films with higher leakage currents. Zhou and Newns proposed a linear mechanism of the dead layer..$^{20}$ Based on Thomas's theory, it has been shown that "dead-layer effects" on the surface of a ferroelectric film can take place without the need for any supposed charge transfer, for example, due to the presence of a Schottky-barrier. These authors do not exclude the additional contribution to the dead layer effect of local electric fields on the film surface. The origin of these electric fields is usually associated with a Schottky-barrier phenomenon, suggesting some charge transfer between the electrode and the film. In this sense, the present work is at least peripheral to some recent studies of "negative capacitance" ${ }^{21}$ and more directly to lozenge-shaped hysteresis loops. ${ }^{22}$

We note peripherally in concluding that our model assumes a large, nearly saturated, domain configuration. For thinner specimens $(\mathrm{d}<75 \mathrm{~nm})$, it is expected that the domain configuration will consist of a larger number of small domains, as shown by Refs. 23 and 24 . Such phenomena also become important nearer Tc, but our studies are $>300 \mathrm{~K}$ below $\mathrm{T}_{\mathrm{C}}$.

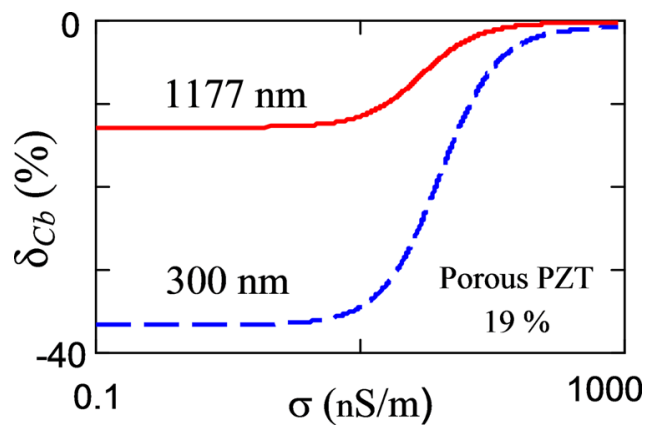

FIG. 4. The dependence of the measurement error of the ferroelectric volume capacitance of the porous PZT film as a function of conductivity that shunts the dead layer on the frequency of $100 \mathrm{~Hz}$.
The dead layer model is used for the analysis of dense and porous thin films. The dead layer thickness $l_{d}$ was calculated from the dielectric portrait slopes that exclude the leakage influence. For comparison, the dead layer thickness was obtained from the dielectric permittivity versus thickness dependence obtained from high frequency small-signal measurements and from slopes of the hysteresis loops at the coercive field point. The small-signal capacitance technique and method based on the dielectric hysteresis portraits give good correlation for dense PZT films: $l_{d} \sim 2.13-2.2 \AA$. For porous PZT films, the dead layer thickness demonstrates higher values: $l_{d}=12 \AA$ for capacitance small-signal measurements and only $l_{d}=5.8 \AA$ for measurements based on the dielectric portrait. This suggests that higher leakage conductivity shunts the electric capacitance of the dead layer with a higher thickness and a lower dielectric constant and therefore reduces its effective thickness. Tagantsev's technique gives six times lower $l_{d}$ values due to the effect of nonlinear leakage currents and relaxation losses.

See supplementary material for a new theoretical model with a small number of experimental examples chosen for illustration of the model from commercial lead zirconate-titanate (PZT) ferroelectrics of different thicknesses and porosities. In order to permit readers to assess the detailed parameters of the experimental specimens, the supplementary information, provided at the request of the editor, gives capacitance, microstructure, ellipsometry, dielectric properties, switched polarization, etc., of all the samples used.

Yu. Podgorny acknowledges the program of Ministry of Education and Science (3.5726.2017/8.9) and K. Vorotilov and A. Sigov thank the program of Ministry of Education and Science (11.2259.2017).

\section{REFERENCES}

${ }^{\text {}}$ B. Chen, H. Yang, L. Zhao, J. Miao, B. Xu, X. G. Qiu, B. R. Zhao, X. Y. Qi, and X. F. Duan, "Thickness and dielectric constant of dead layer in Pt/ $\left(\mathrm{Ba}_{0.7} \mathrm{Sr}_{0.3}\right) \mathrm{TiO}_{3} / \mathrm{YBa}_{2} \mathrm{Cu}_{3} \mathrm{O}_{7-\mathrm{x}}$ capacitor," Appl. Phys. Lett. 84, 583-585 (2004).

${ }^{2}$ A. Q. Jiang, C. Wang, B. L. Cheng, and Z. H. Chen, "Experimental determination of interfacial-layer thickness from polarization-voltage hysteresis loops in $\mathrm{Pb}\left(\mathrm{Zr}_{0.4} \mathrm{Ti}_{0.6}\right) \mathrm{O}_{3}$ thin films," Appl. Phys. Lett. 86, 202904 (2005).

${ }^{3}$ B. T. Lee and C. S. Hwang, "Influences of interfacial intrinsic low-dielectric layers on the dielectric properties of sputtered (Ba,Sr) $\mathrm{TiO}_{3}$ thin films," Appl. Phys. Lett. 77(1), 124-126 (2000).

${ }^{4}$ H. Yang, N. A. Suvorova, M. Jain, B. S. Kang, Y. Li, M. E. Hawley, P. C. Dowden, R. F. DePaula, Q. X. Jiaa, and C. J. Lu, "Effective thickness and dielectric constant of interfacial layers of $\mathrm{Pt} / \mathrm{Bi}_{3.15} \mathrm{Nd}_{0.85} \mathrm{Ti}_{3} \mathrm{O}_{12} / \mathrm{SrRuO}_{3}$ capacitors," Appl. Phys. Lett. 90, 232909 (2007)

${ }^{5}$ P. K. Larsen, G. J. M. Dormans, D. J. Taylor, and P. J. van Veldhoven, "Ferroelectric properties and fatigue of $\mathrm{PbZr}_{0.51} \mathrm{Ti}_{0.49} \mathrm{O}_{3}$ thin films of varying thickness: Blocking layer model," J. Appl. Phys. 76, 2405-2413 (1994).

${ }^{6}$ J. J. Lee, C. L. Thio, and S. B. Desu, "Electrode contacts on ferroelectric $\mathrm{Pb}\left(\mathrm{Zr}_{\mathrm{x}} \mathrm{Ti}_{1-\mathrm{x}}\right) \mathrm{O}_{3}$ and $\mathrm{SrBi}_{2} \mathrm{Ta}_{2} \mathrm{O}_{9}$ thin films and their influence on fatigue properties," J. Appl. Phys. 78, 5073 (1995).

${ }^{7}$ J. Kim, J. Рак, K. Nam, and G. Park, "Ferroelectric $(\mathrm{Ba}, \mathrm{Sr}) \mathrm{TiO}_{3}$ thin films on $\mathrm{Pt} / \mathrm{Ti} / \mathrm{SiO}_{2} / \mathrm{Si}$ substrates by the sol-gel process and evaluation of the intrinsic dead layers," J. Korean Phys. Soc. 47, S349 (2005).

${ }^{8}$ Y. V. Podgorny, D. S. Seregin, A. S. Sigov, and K. A. Vorotilov, "Effect of solgel PZT film thickness on the hysteresis properties," Ferroelectrics 439(1), 74-79 (2012).

${ }^{9}$ A. K. Tagantsev, M. Landivar, E. Colla, and N. Setter, "Identification of passive layer in ferroelectric thin films from their switching parameters," J. Appl. Phys. 78, 2623-2630 (1995).

${ }^{10}$ A. K. Tagantsev and G. Gerra, "Interface-induced phenomena in polarization response of ferroelectric thin films,” J. Appl. Phys. 100, 051607 (2006). 
${ }^{11} \mathrm{U}$. Böttger and R. Waser, "Interaction between depolarization effects, interface layer, and fatigue behavior in PZT thin film capacitors," J. Appl. Phys. 122, 024105 (2017).

${ }^{12}$ M. Dawber, K. M. Rabe, and J. F. Scott, "Physics of thin-film ferroelectric oxides," Rev. Mod. Phys. 77, 1083-1130 (2005).

${ }^{13}$ Yu. Podgorny, K. Vorotilov, P. Lavrov, and A. Sigov, "Effect of leakage and relaxation losses in the determination the hysteresis parameters of the ferroelectric nanostructure," J. Nanomater. Nanostruct. - XXI Century 2(7), 20-29 (2016) (in Russian ISSN 2225-0999).

${ }^{14} \mathrm{~L}$. Jin, F. Li, and S. Zhang, "Decoding the fingerprint of ferroelectrics loops: Comprehension of the material properties and structures," J. Am. Ceram. Soc. 97(1), 1-27 (2014).

${ }^{15}$ N. M. Kotova, K. A. Vorotilov, D. S. Seregin, and A. S. Sigov, "Role of precursors in the formation of lead zirconate titanate thin films," Inorg. Mater. 50(6), 612-616 (2014).

${ }^{16}$ Yu. Podgorny, K. Vorotilov, P. Lavrov, and A. Sigov, "Leakage currents in porous PZT films,” Ferroelectrics 503(1), 77-84 (2016).
${ }^{17}$ S. L. Miller, R. D. Nasby, J. R. Schwank, M. S. Rodgers, and P. V. Dressendorfer, "Device modeling of ferroelectric capacitors," J. Appl. Phys. 68, 6463-6471 (1990).

${ }^{18}$ Y. Podgorny, K. Vorotilov, and A. Sigov, "Estimation of steady-state leakage current in polycrystalline PZT thin films," AIP Adv. 6, 095025 (2016).

${ }^{19}$ S. M. Sze and K. Ng Kwok, Physics of Semiconductor Devices, 3rd ed. (John Wiley and Sons, New York, 2007).

${ }^{20} \mathrm{C}$. Zhou and D. M. Newns, "Intrinsic dead layer effect and the performance of ferroelectric thin film capacitors,” J. Appl. Phys. 82, 3081-3088 (1997).

${ }^{21}$ P. Zubko, J. C. Wojdeł, M. Hadjimichael, S. F. Pena, A. Sené, I. Luḱyanchuk, J.-M. Triscone, and J. Íniguez, Nature 534, 524-527 (2016).

22. F. Scott and J. Gardner, "Ferroelectrics," Mater. Today 21, 553-562 (2018).

${ }^{23}$ A. M. Bratkovsky and A. P. Levanyuk, Phys. Rev. Lett. 84, 3177 (2000).

${ }^{24}$ E. A. Eliseev and A. N. Morozovska, J. Mater. Sci. 44, 5149-5160 (2009).

${ }^{25}$ The polarization dependence of the hysteresis loop tips on the voltage is usually called as the dielectric portrait of the ferroelectric structure. 